\title{
Resistencia química del hormigón XXIII. Influencia de la adición de escoria a un cemento portland resistente al yeso. Estudio de la concentración iónica del sistema cemento 2/escoria-agua potable filtrada
}

\author{
JOSE LUUIS SAGRERA-MORENO y DEMETRIO GASPAR-TEBAR
}

Drs. en Ciencias Quimicas (IETCC/CSIC)

\begin{abstract}
$R E S U M E N$
En el presente trabajo, continuación de otros, se estudia la variación del $\mathrm{pH}$, asi como de la concentración de los iones $\mathrm{Ca}(\mathrm{II})$ y $\mathrm{SO}_{4}(\mathrm{II})$ de los medios (agua potable filtrada) en donde han estado sumergidas las probetas de mortero $(1: 3)$ hechas con un cemento portland industrial resistente al yeso (cemento $2<>P-450-Y$ ) y con las mezclas cemento 2/escoria $=85 / 15-65 / 35-40 / 60$ y 30/70, en peso, durante el periodo de curado (21 dias) 1 , posteriormente, durante $56-90-180$ y60 dias (periodos de conservación), en esta etapa. Asi mismo, se estudia la composición quimica de las nuevas fases sólidas formadas en dichos medios; la composición estructural de dichas fases sólidas se determinó por DRX (calcita, en ambos periodos .; además, aragonito durante el periodo de conservacion de las series de probetas hechas sólo con cemento 2), de ello se dio cuenta en (2).
\end{abstract}

En este sistema cemento 2/escoria-agua potable filtrada se ha puesto de manifiesto que se produce un incremento del contenido de Ca (II) en la disolución y en las mencionadas fases sólidas, asi como del valor del pH, que dependen de la mezcla utilizada para fabricar las diversas series de probetas !' del tiempo de conservación. Las cantidades de Ca (II) que se encuentran en las distintas soluciones (agua potable filtrada) son, normalmente, menores de $0,1 \times 10^{-2}$ moles, mientras que en las nuevas fases sólidas son superiores a dicha cantidad $y$, por regla general, a $0,5 \times 10^{-2}$ moles.

El ion $\mathrm{SO}_{4}$ (II) no se ha detectado en las nuevas fases sólidas, habiéndose encontrado en pequeñas cantidades en las distintas fases liquidas (agua potable filtrada). El contenido de dichos iones es, normalmente, del mismo orden que el del agua potable filtrada en los medios en donde han estado sumergidas las diversas series de probetas, excepto en aquellos medios en donde han estado las probetas que tienen los mayores contenidos de escoria (60) $70 \%$, en peso).

\author{
$S U M M A R Y$
}

In this stud!, which follow's on from others, the $\mathrm{pH}$ rariation is examined and also that of the concentration of $\mathrm{Ca}(\mathrm{II})$ and $\mathrm{SO}_{4}$ (II) ions in the mediums (filtered potable water) in which the test samples of mortar (1:3) made with industrial portland cement resistent to gypsum (cement $2<>P-450-Y$ ) and with the mixtures cement $2 /$ slag $=85 / 15-65 / 35-40 / 60$ and 30/70, in weight, have been submerged throughout the curing period (21 da!s) and, subsequently, for $56-90-180$ and 360 da!'s (preservation periods), at this stage. In the same way, the chemical composition of these solid stages was determined by XRD (calcite, in both peiods and, also, aragonite during the preservation period of the series of test samples made only with cement 2); an account of this was given in (2).

In this cement 2/slag-filtered potable water system, it has been shown that an increase in the content of Ca (II) occurs in dissolution and the solid stages mentioned, and also in the $\mathrm{pH}$ value, depending on the mixture used to make up the different series of test samples and the length of preservation time. The quantities of $\mathrm{Ca}$ (II) found in the different dissolutions (filtered potable water) are normally smaller than $0.1 \times 10^{-2}$ moles, while in the new solid stages they are greater than this quantity and, as a general rule, exceed $0.5 \times 10^{-2}$ moles.

The $\mathrm{SO}_{4}(\mathrm{II})$ ion has not been detected in the new solid stages, but has been found in small quantities in the different liquid stages (filtered potable water). The content of these ions is normally of the same order as that of filtered potable water in those mediums in which the different series of test samples have been submerged, with the exception of those mediums which have had the test samples with greatest slag content ( 60 and $70 \%$, in weight) submerged in them. 


\section{INTRODUCCION}

En otros trabajos anteriores (1) (2) (3) se estudió:

a) La evolución que experimenta la resistencia quimica por el método de Kock-Steinegger y las modificaciones sufridas por las resistencias mecánicas a flexotracción de las probetas de mortero $(1: 3)$ de $1 \times 1 \times 6 \mathrm{~cm}$ hechas con el cemento portland industrial resistente al yeso (cemento $2<>\mathrm{P}-450-\mathrm{Y}$ ), objeto del presente artículo, y con las mezclas cemento 2/escoria $=85 / 15-65 / 35-40 / 60$ y $30 / 70$, en peso, sumergidas en agua potable filtrada $\left({ }^{*}\right)$, unas series de 12 probetas, y en agua de mar artificial ASTM (D 1141-75) (**), otras series análogas de 12 probetas, durante 56 - 90 - 180 y 360 dias, en esta etapa, después de haberlas curado 24 horas en un recinto saturado de humedad y, a continuación, bajo agua potable filtrada hasta 22 dias (1).

b) La composición estructural de las nuevas fases sólidas formadas en los medios de curado y de conservación (agua potable filtrada) (2), asi como de conservación-ataque (agua de mar artificial ASTM) (3), en donde han estado sumergidas las mencionadas series de probetas de mortero.

c) La evolución de las características estructurales de la fracción enriquecida (cemento 2 hidratado y cemento 2 hidratado-atacado) extraida de uno de los prismas de mortero de cada serie de probetas de mortero de los sistemas cemento 2 /escoria-agua potable filtrada (2) y cemento 2/escoria-agua de mar artificial ASTM (3).

En el presente artículo, continuación de los anteriores y que se ampliará con otros, se estudia la variación de la concentración de los iones calcio y sulfato en los medios de curado y de conservación (agua potable filtrada) en donde han estado sumergidas las diversas series de probetas de mortero, asi como de las fases sólidas formadas, y la evolución del $\mathrm{pH}$ de dichos medios.

\section{PARTE EXPERIMENTAL}

Con el fin de conocer la variación de la concentración de los iones calcio y sulfato, así como del $\mathrm{pH}$, del agua potable filtrada en donde han estado sumergidas las probetas de mortero $(1: 3)$, hechas con cemento 2 y con las mezclas cemento 2 /escoria $=85 / 15-65 / 35-40 / 60$ y $30 / 70$ (en peso), durante el período de curado (21 días) y de conservación $(56,90,180$ y 360 días) se ha separado, en una primera etapa, la fase sólida formada, que se ha secado en una corriente de nitrógeno; posteriormente, se ha determinado la concentración de los iones señalados y el valor del $\mathrm{pH}$.

En la nueva fase sólida, puesta en disolución por ataque con una disolución de ácido clorhidrico diluido $(1+1)$, se ha determinado el contenido de $\mathrm{Ca}$ (II) y de $\mathrm{SO}_{4}$ (II).

El contenido de los iones calcio se ha determinado complexométricamente con EDTA, el de los iones sulfato gravimétricamente al estado de sulfato de bario, y el valor del $\mathrm{pH}$ por medio de un pH-metro, marca Crison, modelo 501.

De los materiales utilizados y características (cemento 2, escoria y arena), de la técnica operatoria seguida para preparar las mezclas de cemento $2 /$ escoria, asi como para curar y conservar las probetas de mortero de las diversas series en agua potable filtrada se dio cuenta en (1).

(*) Sistema: cemento $2 /$ escoria-agua potable filtrada.

(**) Sistema: cemento 2 /escoria-agua de mar artificial. 


\section{RESULTADOS Y DISCUSION}

\subsection{Período de curado}

En la tabla 1 figuran las cantidades de Ca (II), en moles $\times 10^{-2}$, que se encuentran en la fase liquida (agua potable filtrada) en donde han estado sumergidas, durante el período de curado (21 días), las diversas series de probetas de mortero, así como el valor del $\mathrm{pH}$. También se encuentran, en dicha tabla, las cantidades del mencionado ion correspondientes a las nuevas fases sólidas formadas en estas condiciones.

En el agua potable filtrada en donde han estado sumergidas las diversas series de probetas de mortero hechas con cemento $2 \mathrm{y}$ con las mezclas cemento $2 /$ escoria $=85 / 15,65 / 35,40 / 60$ y $30 / 70$ (en peso) durante el período de curado (21 días) se ha producido un incremento de $\mathrm{Ca}$ (II), que se ha detectado en la disolución y en la nueva fase sólida, constituida por el compuesto cristalino calcita como se ha puesto de manifiesto en (2).

El contenido de $\mathrm{Ca}$ (II) en el agua potable filtrada es $0,005 \mathrm{~g} / l<>0,0025 \mathrm{~g}$ en $500 \mathrm{~m} l<>0,006 \times$ $\times 10^{-2}$ moles; dicho contenido en la mencionada agua en donde se han curado las diversas series de probetas, superior al anterior, es función de la mezcla utilizada para fabricar las mencionadas series. El valor más alto $\left(0,23 \times 10^{-2}\right.$ moles $)$ corresponde al medio en donde han estado las probetas hechas con cemento 2, disminuyendo para los casos restantes (medios en donde han estado las probetas elaboradas con las distintas mezclas cemento 2 /escoria), tabla 1 y figura 1.

TABLA 1

Sistema: cemento 2/escoria-agua potable filtrada

Evolución de los contenidos de Ca (II) y del pH durante el período de curado (21 días)

\begin{tabular}{|c|c|c|c|c|}
\hline \multirow{2}{*}{$\begin{array}{c}\text { Mezcla: } \\
\text { cemento 2/escoria } \\
\text { (en peso) }\end{array}$} & $\begin{array}{c}\text { Case liquida } \\
\text { (Disolución) }\end{array}$ & Fase sólida & Suma & pH \\
\cline { 2 - 5 } & 0,23 & 1,22 & 1,45 & 11 \\
\hline $100 / 0$ & 0,08 & 1,57 & 1,65 & 11,5 \\
$85 / 15$ & 0,06 & 1,32 & 1,38 & 11,5 \\
$65 / 35$ & 0,06 & 1,40 & 1,46 & 9,5 \\
$40 / 60$ & $0,00_{4}$ & 0,88 & 0,89 & 9,5 \\
$30 / 70$ & \multicolumn{2}{|c|}{} & & \\
\hline
\end{tabular}

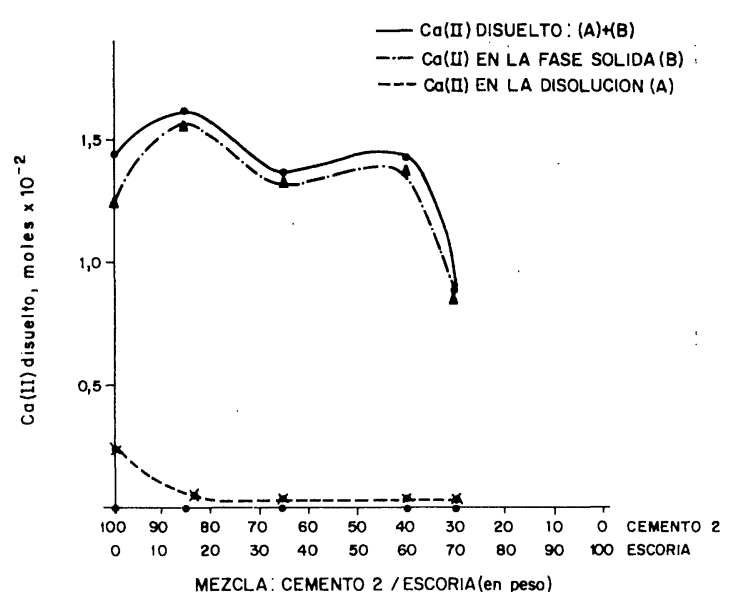

Fig. 1.--Sistema: cemento 2/escoria-agua potable filtrada. Periodo de curado. Evolución del contenido de Ca (II) disuelto.
En dichas tabla y figura se observa que, prácticamente, todo el $\mathrm{Ca}$ (II) se encuentra en la fase sólida formando parte de la calcita, en cantidades superiores a las teóricas que aportarían las diversas mezclas cemento 2/escoria utilizadas en este sistema, suponiendo inerte a la escoria, desde este punto de vista.

El pH del agua potable filtrada $(7,4)$ se ha incrementado en todos los casos, alcanzando los valores más altos en los medios en donde han estado sumergidas las probetas de mortero hechas con cemento 2 y con las mezclas que tienen 15 y $35 \%$ de escoria (en peso) y los más bajos, del mismo orden, en donde se han curado las pro- 
betas fabricadas con las mezclas cemento $2 /$ escoria $=40 / 60$ y $30 / 70$ (en peso).

La nueva fase sólida formada, impurificada a veces por pequeñas fracciones desprendidas de las probetas de mortero (2), sigue una evolución análoga a la de las cantidades de $\mathrm{Ca}$ (II) de dicha fase sólida.

\subsection{Periodo de conservación}

En el medio (agua potable filtrada) en donde han estado sumergidas las distintas series de probetas de mortero, después del período de curado, durante $56-90-180$ y 360 días se ha producido un incremento de la concentración de iones $\mathrm{Ca}$ (II), $\mathrm{SO}_{4}$ (II)-suave, asi como del valor del $\mathrm{pH}$, habiéndose formado una nueva fase sólida, que está constituida por el compuesto cristalino calcita en todos los casos y, además, por aragonito la fase que ha aparecido en el agua potable filtrada en donde han estado las diversas series de probetas fabricadas con cemento 2 sin adición de escoria, como se probó en (2).

En la tabla 2 figuran los resultados obtenidos, para cada edad y para cada serie de probetas de mortero, correspondientes a las nuevas fases sólidas formadas [cantidad, compuestos cristalinos

TABLA 2

Sistema:cemento 2/escoria-agua potable filtrada

Evolución de la fase sólida formada, del $\mathrm{pH} y$ de los contenidos de $\mathrm{Ca}$ (II) y de $\mathrm{SO}_{4}$ (II)

\begin{tabular}{|c|c|c|c|c|c|c|c|c|c|c|}
\hline \multirow{2}{*}{$\begin{array}{l}\text { Cemento } 2 / \\
\text { (en peso) }\end{array}$} & \multirow{2}{*}{$\begin{array}{r}\text { Edad } \\
\text { dias }\end{array}$} & \multicolumn{2}{|c|}{ Fase sólida } & \multirow{2}{*}{$\underset{\text { pH }}{\text { Disolución }}$} & \multicolumn{3}{|c|}{ Ca (II); moles $\times 10^{-2}$} & \multicolumn{3}{|c|}{$\mathrm{SO}_{4}(\mathrm{II}) ;$ moles $\times 10^{-2}$} \\
\hline & & $\mathbf{g}$ & DRX & & Disolución & Fase sólida & Suma & Disolución & Fase sólida & Suma \\
\hline $100 / 00$ & & 1,12 & $\mathrm{Cc}+\mathrm{Ag}$ & 12,0 & 0,11 & 1,12 & 1,23 & 0,01 & n.d. & 0,01 \\
\hline $85 / 15$ & & 0,92 & Calcita & 12,2 & 0,04 & 0,82 & 0,86 & $0,00_{4}$ & n.d. & $0,00_{4}$ \\
\hline $65 / 35$ & 56 & 0,67 & $"$ & 10,1 & 0,04 & 0,60 & 0,64 & $0,00_{4}$ & n.d. & $0,00_{4}$ \\
\hline $40 / 60$ & & 0,48 & 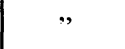 & 8,6 & 0,16 & 0,25 & 0,41 & 0,01 & n.d. & 0,01 \\
\hline $30 / 70$ & & 0,11 & $"$ & 8,5 & 0,04 & 0,10 & 0,14 & 0,02 & n.d. & 0,02 \\
\hline $100 / 0$ & & 1,82 & $\mathrm{Cc}+\mathrm{Ag}$ & 8,7 & 0,04 & 1,65 & 1,69 & 0,02 & n.d. & 0,02 \\
\hline $85 / 15$ & & 1,50 & Calcita & 8,6 & 0,04 & 1,35 & 1,39 & $0,00_{6}$ & n.d. & $0,00_{6}$ \\
\hline $65 / 35$ & 90 & 0,59 & $"$ & 8,6 & 0,03 & 0,45 & 0,48 & $0,00_{8}$ & n.d. & $0,00_{8}$ \\
\hline $40 / 60$ & & 0,50 & $"$ & 8,5 & 0,03 & 0,42 & 0,45 & 0,02 & n.d. & 0,02 \\
\hline $30 / 70$ & & 0,39 & $"$ & 8,3 & 0,04 & 0,35 & 0,39 & 0,03 & n.d. & 0,03 \\
\hline $100 / 0$ & & 1,86 & $\mathrm{Cc}+\mathrm{Ag}$ & 12,1 & 0,54 & 1,65 & 2,19 & 0,01 & n.d. & 0,01 \\
\hline $85 / 15$ & & 1,56 & Calcita & 10,0 & 0,02 & 1,67 & 1,69 & $0,00_{3}$ & n.d. & $0,00_{3}$ \\
\hline $65 / 35$ & 180 & 0,89 & $"$ & 8,7 & 0,02 & 0,82 & 0,84 & $0,00_{9}$ & n.d. & $0,00_{9}$ \\
\hline $40 / 70$ & & 0,69 & $"$ & 7,9 & 0,05 & 0,70 & 0,75 & $0,00_{6}$ & n.d. & $0,00_{6}$ \\
\hline $30 / 70$ & & 0,60 & $"$ & 8,4 & 0,06 & 0,52 & 0,58 & 0,05 & n.d. & 0,05 \\
\hline $100 / 0$ & & 3,17 & $\mathrm{Cc}+\mathrm{Ag}$ & 11,3 & 0,04 & 2,87 & 2,91 & $0,00_{7}$ & n.d. & $0,00_{7}$ \\
\hline $85 / 15$ & & 2,17 & Calcita & 8,1 & 0,02 & 2,05 . & 2,07 & 0,01 & n.d. & 0,01 \\
\hline $65 / 35$ & 360 & 1,80 & $"$ & 8,0 & 0,04 & 1,65 & 1,69 & 0,04 & n.d. & 0,04 \\
\hline $40 / 60$ & & 1,17 & $"$ & 8,1 & 0,08 & 1,05 & 1,13 & 0,09 & n.d. & 0,09 \\
\hline $30 / 70$ & & 0,55 & $"$ & 7,9 & 0,10 & 0,50 & 0,60 & 0,15 & n.d. & 0,15 \\
\hline
\end{tabular}

n.d. $=$ no detectado; $\mathrm{Cc}=$ Calcita; $\mathrm{Ag}=$ Aragonito. 
identificados por DRX, contenidos de $\mathrm{Ca}$ (II) y de $\mathrm{SO}_{4}$ (II)] y a las disoluciones, agua potable filtrada, [pH, cantidades de $\mathrm{Ca}$ (II) y de $\mathrm{SO}_{4}$ (II)] en donde se han conservado dichas series de probetas.

\section{Evolución de la fase sólida}

En las figuras 2 y 3 se ha representado la evolución de la fase sólida en función de la mezcla cemento 2/escoria utilizada en la fabricación de la diversas series de probetas de mortero para cada edad, en la primera, y del tiempo de conservación para cada mezcla, en la segunda.

La cantidad de fase sólida que se produce en los medios de conservación de las distintas series de probetas de mortero es función, para cada edad, de la mezcla utilizada en la fabricación de las mencionadas series de probetas (figura 2), experimentando una disminución conforme lo hace la cantidad de cemento 2 en la mezcla; esta disminución es más acentuada según aumenta el tiempo de conservación. Dicha cantidad de fase sólida, para cada mezcla, depende del tiempo de cnservación (figura 3), aumentando, por regla general, conforme lo hace la edad.
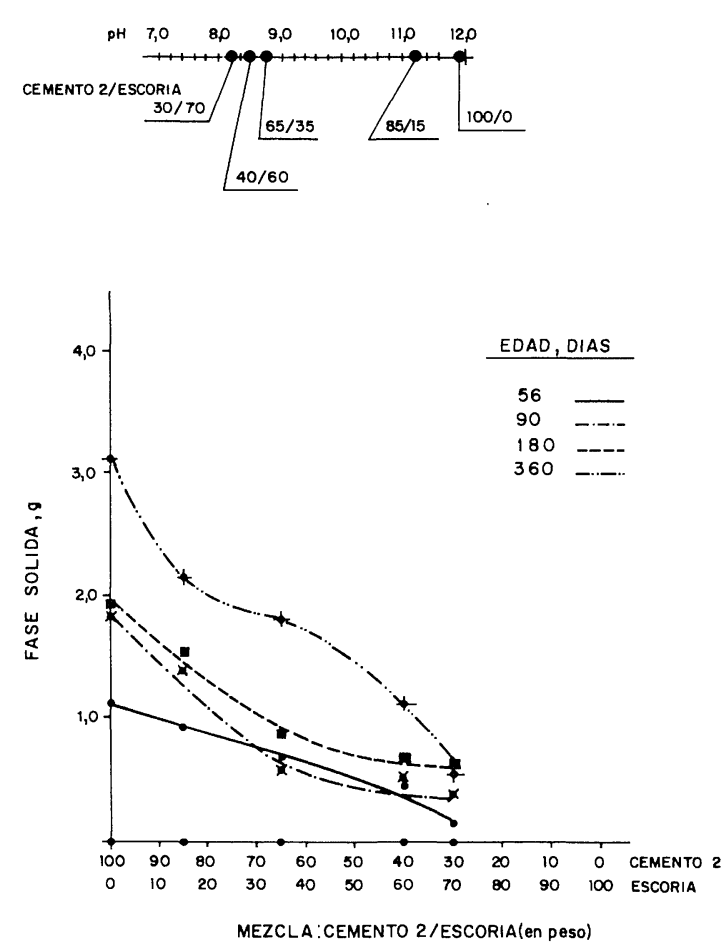

Fig. 2.- Sistema: cemento 2/escoria-agua potable filtrada. Evolución de la cantidad de fase sólida y del $\mathrm{pH}$.
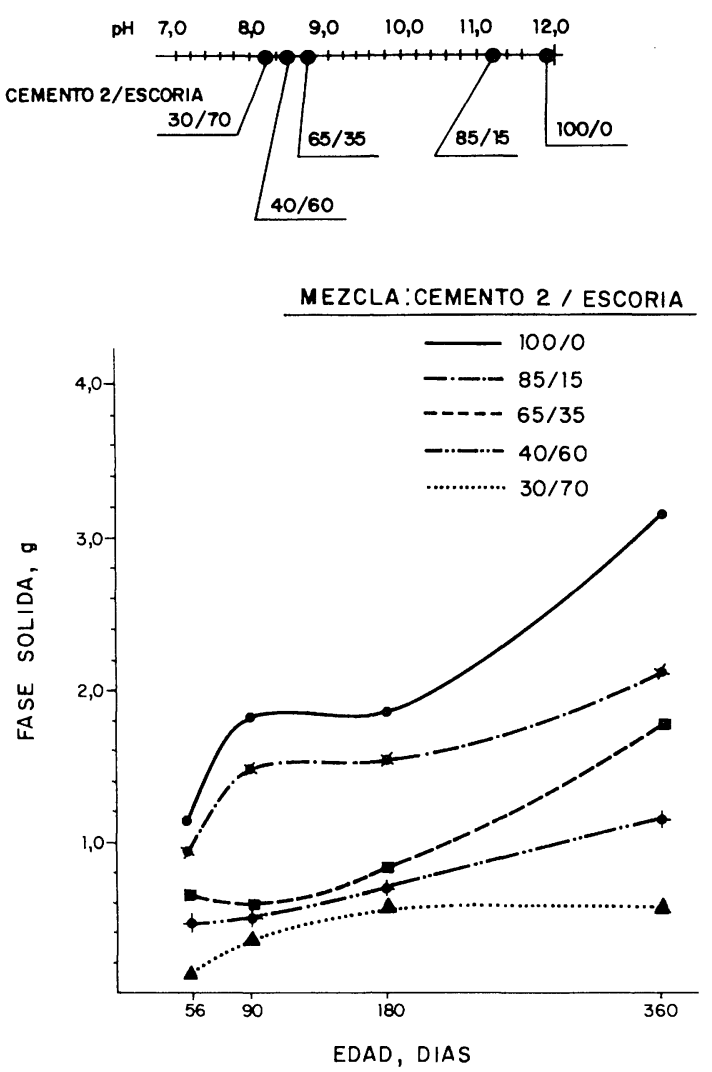

Fig. 3.-Sistema: cemento 2/escoria-agua potable filtrada. Evolución de la cantidad de fase sólida y del $\mathrm{pH}$.

Los incrementos experimentados en la fase sólida formada en los medios en donde han estado sumergidas las probetas durante 360 días, con relación a las conservadas durante 56 dias, han sido $2,05-1,25-1,13-0,69$ y $0,44 \mathrm{~g}$ para las series hechas con cemento $2 \mathrm{y}$ con las mezclas cemento $2 /$ escoria $=85 / 15,65 / 35,40 / 60$ y $30 / 70$ (en peso), respectivamente.

\section{Evolución del contenido de Ca (II)}

En las figuras 4 y 5 se ha representado la evolución del contenido de Ca (II) que existe en la 
disolución y en la nueva fase sólida [Ca (II) procedente de los compuestos del cemento disueltos], junto con el $\mathrm{Ca}$ (II) presente en $\operatorname{los} 800 \mathrm{ml}$ de agua potable filtrada, en función de la mezcla cemento 2/escoria utilizada en la fabricación de las distintas series de probetas de mortero, primera figura, y del tiempo que han estado sumergidas las mencionadas series de probetas de mortero en agua potable filtrada, en la segunda figura.

El contenido de Ca (II) del agua potable filtrada $\left(0,005 \mathrm{~g} / l<>0,004 \mathrm{~g} / 800 \mathrm{~m} l<>0,01 \times 10^{-2}\right.$ moles) se ha incrementado en todos los casos (tabla 2). Las cantidades de $\mathrm{Ca}$ (II) en las disoluciones en donde han estado sumergidas las distintas series de probetas son superiores a $0,01 \times 10^{-2}$ moles e inferiores a $0,1 \times 10^{-2}$ moles en todos los casos, excepto para los medios en donde han estado las series de probetas hechas con cemento 2 para $t=22+56$ y $22+180$ días, y con las mezclas cemento $2 /$ escoria $=40 / 60$ y 30/70 (en peso) para $t=22+56$ días y $22+360$ dias, respectivamente. Por el contrario, en las nuevas fases sólidas son superiores a dicha cantidad y, normalmente, a $0,5 \times 10^{-2}$ moles.

En la figura 4 se observa que dicha cantidad de $\mathrm{Ca}$ (II) total [Ca (II) en la disolución $+\mathrm{Ca}$ (II) en la nueva fase sólidal es función, para cada edad, de la mezcla utilizada en la fabricación de las distintas series de probetas experimentando una disminución conforme lo hace el contenido de cemento en dichas mezclas. Esta disminución es tanto más acentuada conforme aumenta el tiempo de conservación (tabla 2); así, pasa de $1,23 \times 10^{-2}$ moles a $0,14 \times 10^{-2}$ moles, para $t=22+56$ dias, y de $2,91 \times 10^{-2}$ moles a $0,60 \times 10^{-2}$ moles, para $t=22+360$ dias, según se trate de los medios en donde han estado sumergidas las probetas de mortero hechas con las mezclas cemento $2 /$ escoria $=100 / 0$ y 30/70 (en peso), respectivamente. La evolución del mencionado contenido de $\mathrm{Ca}$ (II) está intimamente ligada a la cantidad de la nueva fase sólida formada (figura 2).

En la figura 5, se aprecia que las cantidades de Ca (II), que existen en la disolución más en la nueva fase sólida, correspondientes a las diversas series de probetas de mortero fabricadas con distintas mezclas, son función del tiempo de conservación para una misma mezcla. Las mayores cantidades de $\mathrm{Ca}$ (II) puestas en juego corresponden, para todas las edades, a los medios en donde han estado sumergidas las series de probetas hechas con cemento 2 y las menores para aquellos medios en donde se han conservado las series de probetas elaboradas con la mezcla que tiene el mayor contenido de escoria ( $70 \%$, en peso).

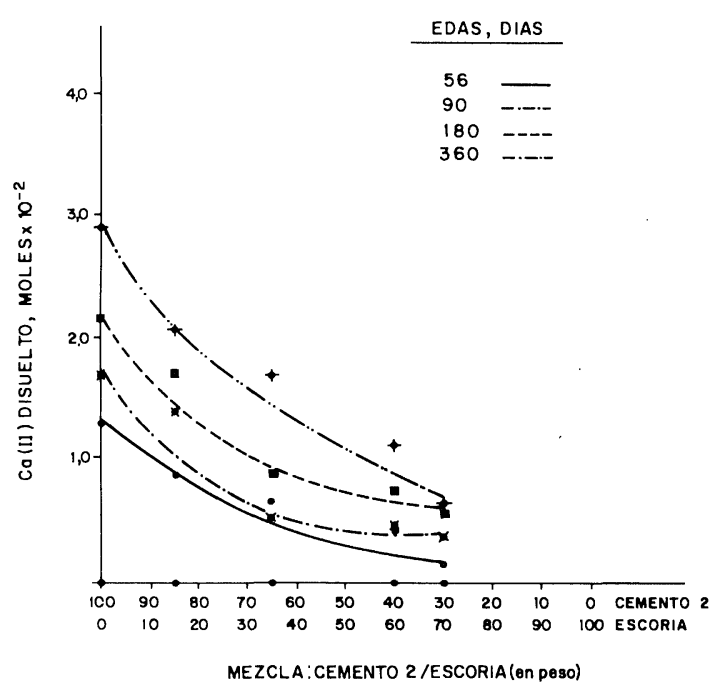

Fig. 4.-Sistema: cemento 2/escoria-agua potable filtrada Fvolución del contenido de Ca (II) disuelto.

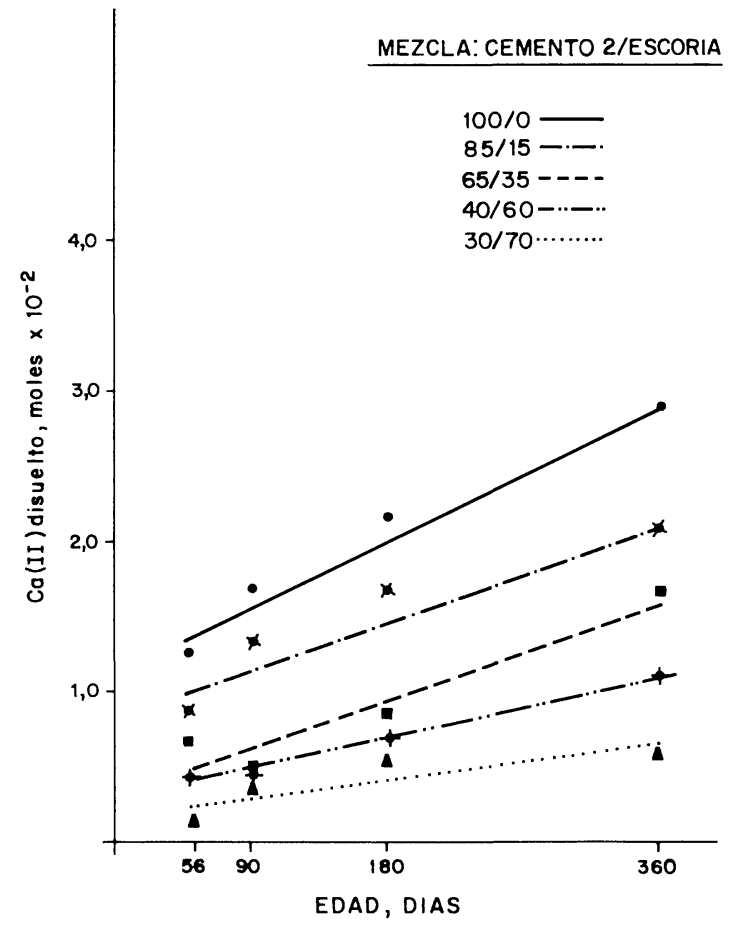

Fig. 5.-. Sistema: cemento $2 /$ escoria-agua potable filtrada. Fvolución del contenido de Ca (II) disuclto. 
Los iones sulfato, únicamente, se han detectado en el agua potable filtrada en donde han estado sumergidas las distintas series de probetas de mortero $(1: 3)$ y no en las nuevas fases sólidas (tabla 2). El contenido de dichos iones en el agua potable filtrada es $0,003 \mathrm{~g} / l<>0,002 \times 10^{-2}$ moles en $800 \mathrm{ml}$ y en los mencionados medios es del mismo orden o ligeramente superior; así, son del mismo orden en los medios en donde han estado sumergidas las series de probetas hechas con las mezclas cemento $2 /$ escoria $=100 / 0$ (en peso) para $t=22+360$ dias; $85 / 15$ (en peso) para $t=22+56,22+90$ y $22+180$ dias; $65 / 35$ (en peso) para $t=22+56$, $22+90$ y $22+180$ dias y $40 / 60$ (en peso) para $t=22+180$ dias. En los casos restantes es igual o superior a $0,01 \times 10^{-2}$ moles.

\section{INTERPRETACION DE RESULTADOS}

El Ca (II) presente en el agua potable filtrada y en la nueva fase sólida del sistema cemento 2 /escoria-agua potable filtrada procede, fundamentalmente, de la disolución del hidróxido de calcio generado en las reacciones de hidratación del cemento, según:

$$
\mathrm{Ca}(\mathrm{OH})_{2} \text {. sólido } \stackrel{\mathrm{H}_{2} \mathrm{O}}{\Longrightarrow} \mathrm{Ca}(\mathrm{OH})_{2} \text {.disuelto } \stackrel{\mathrm{H}_{2} \mathrm{O}}{=} \mathrm{Ca}(\mathrm{II})+2 \mathrm{OH}(\mathrm{I})
$$

produciéndose, además, un aumento de la concentración de iones $\mathrm{OH}$ (I) y, por consiguiente, del valor del $\mathrm{pH}$.

La solubilidad del $\mathrm{Ca}(\mathrm{OH})_{2}$ en agua, a $20^{\circ} \mathrm{C}$, es $1,230 \mathrm{~g} / \mathrm{l}(4)<>1,66 \times 10^{-2} \mathrm{moles} / \mathrm{li}-$ tro $<>1,33 \times 10^{-2}$ moles en $800 \mathrm{ml}$; cantidad que no se ha alcanzado en el Ca (II) presente en la disolución más en la nueva fase sólida de aquéllos medios en donde han estado sumergidas las diversas series de probetas hechas con cemento 2 y con todas las mezclas cemento 2 /escoria sumergidas durante 56 dias, con las mezclas cemento $2 /$ escoria $=65 / 35,40 / 60$ y $30 / 70$ (en peso) para las edades correspondientes a 90 y 180 dias, y 360 días para las dos últimas mezclas. En las distintas disoluciones las cantidades de $\mathrm{Ca}$ (II) son menores de $0,6 \times 10^{-2}$ moles y, normalmente, inferiores a $0,1 \times 10^{-2}$ moles, habiendo precipitado en gran parte como $\mathrm{CaCO}_{3}$ según la reacción (B), por lo que en estos medios puede continuar la reacción (A) de disolución del $\mathrm{Ca}(\mathrm{OH})_{2}$, si se dan condiciones favorables.

El $\mathrm{CO}_{2}$ disuelto en el agua reacciona, en medio básico fuerte, con el $\mathrm{Ca}$ (II) presente en la disolución, de acuerdo con la siguiente reacción:

$$
\mathrm{CO}_{2}+\mathrm{Ca}(\mathrm{II})+2 \mathrm{OH}(\mathrm{I}) \rightleftharpoons \mathrm{CaCO}_{3} \text {. sólido }+\mathrm{H}_{2} \mathrm{O}
$$

dando lugar a la formación de la nueva fase sólida, a una disminución de la concentración de iones $\mathrm{OH}$ (I) del medio y, por consiguiente, del valor del $\mathrm{pH}$, facilitando la solubilidad de la portlandita y favoreciendo las reacciones de hidratación de los compuestos del cemento; el hidróxido de calcio pasa, en parte, a la disolución en forma iónica, según la reacción (A), a la vez que los silicatos correspondientes se degradan.

Para que la reacción de carbonatación tenga lugar, en las condiciones de este sistema lelevada concentración de $\mathrm{OH}$ (I), cantidades de Ca (II) que oscilan entre $0,14 \times 10^{-2}$ moles y $2,91 \times 10^{-2} \mathrm{moles} / 800 \mathrm{ml}<>0,18 \times 10^{2}$ moles litro y $3,6 \times 10^{2} \mathrm{moles} /$ litrol se debe cumplir:

$$
|\mathrm{Ca}(\mathrm{II})|\left|\mathrm{CO}_{3}(\mathrm{II})\right|>P_{\mathrm{s}}\left(4,8 \times 10^{9}\right)
$$

por lo que, teóricamente, en estos casos se precisan cantidades de $\mathrm{CO}_{2}$ comprendidas entre $26,7 \times 10^{-7} \mathrm{moles} /$ litro y $1,3 \times 10^{7} \mathrm{moles} / \mathrm{litro}$. 
La escoria que existe en la mezcla fija, parcialmente, el $\mathrm{Ca}(\mathrm{OH})_{2}$ naciente formando compuestos de calcio, dando lugar a la disminución de unos y al incremento de otros. La fracción correspondiente al clínker es la primera que reacciona con el agua formando los compuestos hidratados del cemento y el hidróxido de calcio, que activa a la fracción escoria, reaccionando con ella y disminuyendo, progresivamente, conforme aumenta la cantidad de escoria; en determinados casos, la reacción es completa (disolución de la portlandita y formación de compuestos con la escoria) no existiendo, por consiguiente, portlandita en la fracción extraída de las probetas de mortero, como se probó en (2).

La cantidad de fase sólida formada es función, para una misma edad, de la mezcla utilizada y, para una mezcla, del tiempo de conservación; esta cantidad depende del $\mathrm{Ca}(\mathrm{OH})_{2}$ disuelto, que está intimamente ligada a la generada en la reacción de hidratación y que no haya formado compuestos poco solubles en agua, más estables que el $\mathrm{Ca}(\mathrm{OH})_{2}$.

En los valores del $\mathrm{pH}$ influye la cantidad de $\mathrm{Ca}(\mathrm{OH})_{2}$ disuelto en el agua potable filtrada, por una parte, que proporciona los iones $\mathrm{OH}(\mathrm{I})$, y del grado de carbonatación, por otra, que en la reacción correspondiente consume dos moles de $\mathrm{OH}$ (I) por cada mol que se forme de $\mathrm{CaCO}_{3}$.

El incremento de la concentración de iones sulfato, que se ha producido en determinados casos $\mathrm{y}$, fundamentalmente, en los medios en donde han estado sumergidas las diversas series de probetas que tienen los mayores contenidos de escoria (60 y $70 \%$, en peso), procede de la disolución de los compuestos correspondientes que se encuentran en el cemento 2 [tiene un contenido de $\mathrm{SO}_{4}$ (II) del $2,8 \%$, en pesol ya que en la escoria no se han detectado, hecho que se pretende demostrar en otros trabajos, como se señaló en (5).

\section{CONCLUSIONES}

\subsection{Período de curado}

\section{Primera:}

En el agua potable filtrada en donde han estado sumergidas las diversas series de probetas de mortero $(1: 3)$, hechas con cemento 2 y con las mezclas cemento 2 /escoria estudiadas en este trabajo, durante 21 dias (periodo de curado), se ha producido un incremento de $\mathrm{Ca}$ (II) que, prácticamente, ha precipitado como $\mathrm{CaCO}_{3}$, en forma de calcita, dando lugar a una nueva fase sólida. Asi mismo, se ha producido un aumento del valor del $\mathrm{pH}$.

Tanto las cantidades de $\mathrm{Ca}$ (II) mencionadas, como los valores del $\mathrm{pH}$, son función de la mezcla cemento 2 /escoria utilizada en la fabricación de las probetas.

\subsection{Período de conservación}

\section{Segunda:}

En el agua potable filtrada en donde han estado sumergidas las distintas series de probetas de mortero, después del periodo de curado, durante $56-90-180$ y 360 dias, se produce un incremento de la concentración de iones calcio y sulfato (suave, la de este último), así como del valor del $\mathrm{pH}$, apareciendo una nueva fase sólida, que está formada por el compuesto cristalino calcita, en todos los casos, y además por aragonito en el medio de conservación en donde han estado sumergidas las probetas hechas con cemento 2 sin adición de escoria. 


\section{Tercera:}

Las cantidades de $\mathrm{Ca}$ (II) en la disolución (agua potable filtrada) en donde han estado sumergidas las diversas series de probetas son, por regla general, menores de $0,1 \times 10^{-2}$ moles y superiores en la nueva fase sólida a $0,5 \times 10^{-2}$ moles, en la mayoría de los casos estudiados.

\section{Cuarta:}

Tanto las cantidades de las nuevas fases sólidas, como las de Ca (II) en la disolución más en la fase sólida, son función, para cada edad, de la mezcla utilizada en la fabricación de las probetas y, para cada mezcla, del tiempo de conservación. Dichas cantidades experimentan una disminución o un incremento conforme es menor la cantidad de cemento 2 en la mezcla empleada o aumenta el tiempo de conservación, respectivamente.

\section{Quinta:}

En la nueva fase sólida no se han detectado los iones sulfato y si en las disoluciones (agua potable filtrada) en donde han estado sumergidas las diversas series de probetas, habiendo experimentado un ligero incremento, con relación al contenido del agua potable filtrada original, en los medios correspondientes a las series de probetas elaboradas con las mezclas que tienen los mayores contenidos de escoria (60 y $70 \%$, en peso).

\section{BIBLIOGRAFIA}

(1) GASPAR-TEBAR, D. y SAGRERA-MORENO, J. L. (1980): Resistencia quimica del hormigón: V. Acción del agua de mar: influencia de la adición de escoria a un cemento portland resistente al yeso. Evolución de las resistencias mecánicas a flexotracción y del coeficiente de corrosión. Materiales de Construcción, julio-agosto-septiembre. 179, 59-79.

(2) SAGRERA-MORENO, J. L. y GASPAR-TEBAR, D. (1981): Resistencia quimica del hormigon: X. Influencia de la adición de escoria a un cemento portland resistente al yeso. Estudio por DRX del sistema cemento 2/escoria-agua potable filtrada. Materiales de Construcción, abril-mayo-junio, 1982, 11-22.

(3) GASPAR-TEBAR, D. y SAGRERA-MORENO, J. L. (1982): Resistencia quimica del hormigón: XVIII. Acción de agua de mar: influencia de la adición de escoria a un cemento portland resistente al yeso. Estudio por DRX. Materiales de Construcción, octubre-noviembre-diciembre, 188, 37-58.

(4) BICZOK, I. (1972): Corrosión y Protección del Hormigón. Ed. Urmo, Bilbao, pág. 211

(5) SAGRERA-MORENO, J. L. y GASPAR-TEBAR, D. (1983): Resistencia quimica del hormigón: XXI. Influencia de la adición de escoria a un cemento portland de alta resistencia inicial. Estudio de la concentración iónica del sistema cemento 1/escoria-agua potable filtrada. Materiales de Construcción, enero-febrero-marzo, 192, 33-41.

\section{RECONOCIMIENTO}

Nuestro más sincero reconocimiento a las personas del equipo de Durabilidad del IETcc: Amalia Rodriguez Pereira, Lucila López Solana, Felipe Cantero Palacios y Manuel Cantero Palacios por su valiosa colaboración en la realización de este trabajo. 